\title{
The Effect of Simultaneous Antigravity Treadmill Training and Electrical Muscle Stimulation After Total Hip Arthroplasty: Short Follow-Up Time
}

\author{
Yulkio Mikami, $\mathrm{MD}, \mathrm{PhD}^{1}$, Naoya Orita, $\mathrm{RPT}^{2}$, Takuma Yamasalki, $\mathrm{MD}, \mathrm{PhD}^{3}$, \\ Yoshiichiro Kamijo, $\mathrm{MD}, \mathrm{PhD}^{4}$, Hiroaki Kimura, $\mathrm{MD}, \mathrm{PhD}^{1}$, Nobuo Adachi, $\mathrm{MD}, \mathrm{PhD}^{3}$ \\ ${ }^{1}$ Department of Rehabilitation Medicine, Hiroshima University Hospital, Hiroshima; \\ ${ }^{2}$ Division of Rehabilitation, Department of Clinical Practice and Support, Hiroshima University Hospital, Hiroshima; \\ ${ }^{3}$ Department of Orthopaedic Surgery, Graduate School of Biomedical Sciences, Hiroshima University, Hiroshima; \\ ${ }^{4}$ Department of Rehabilitation Medicine, Wakayama Medical University, Wakayama, Japan
}

Objective To assess the effectiveness of our devised hybrid physiotherapy regime using an anti-gravity treadmill and a low-frequency electrical stimulation device, as measured in patients with hip osteoarthritis after total hip arthroplasty (THA).

Methods The outcomes of the postoperative rehabilitation in 44 patients who underwent THA for hip osteoarthritis were retrospectively examined. The conventional group $(\mathrm{n}=22)$ underwent the postoperative rehabilitation according to our protocol, while the hybrid group $(\mathrm{n}=22)$ underwent the same training, along with training on an anti-gravity treadmill and training using a low-frequency therapeutic device. The outcome measures were recorded and reviewed with the Numerical Rating Scale for pain, which rates pain on an 11-point scale from 0 to 10 , surgical side knee joint extension force, 10-m walking test, Timed Up and Go test, and the 6-minute walking distance (6MD). The outcome measurement was taken 2 weeks after conducting pre-operation and antigravity treadmill training and electrical muscle stimulation, and compared the respective results.

Results At the timeframe of 2 weeks from the surgery after conducting a devised hybrid physiotherapy, the values of knee extension muscle strength and 6MD were not worse in the hybrid group than conventional group. In the evaluation at 2 weeks after surgery, the knee extension muscle strength and $6 \mathrm{MD}$ values significantly decreased compared with the preoperative values only in the conventional group.

Conclusion Lower limb muscular strength and endurance were maintained in the hybrid group, which suggested that hybrid physiotherapy could maintain physical functions early after THA operation.

Keywords Anti-gravity treadmill, Low frequency electrical stimulation device, Hip osteoarthritis, Total hip arthroplasty

Received October 29, 2018; Accepted January 28, 2019

Corresponding author: Yukio Mikami

Department of Rehabilitation Medicine, Hiroshima University Hospital, Kasumi 1-2-3, Minamiku, Hiroshima 734-8551, Japan. Tel: +81-82-257-5566; Fax: +81-82-257-5594, E-mail: ymikami@wakayama-med.ac.jp

*Current affiliation: Wakayama Medical University, Wakayama, Japan (E-mail: ymikami@wakayama-med.ac.jp)

ORCID: Yukio Mikami (http://orcid.org/0000-0001-6855-8439); Naoya Orita (http://orcid.org/0000-0001-5402-4874); Takuma Yamasaki (http://orcid. org/0000-0002-5848-2079); Yoshiichiro Kamijo (http://orcid.org/0000-0002-4368-1227); Hiroaki Kimura (http://orcid.org/0000-0003-0005-5805); Nobuo Adachi (http://orcid.org/0000-0002-5570-3729).

(a) This is an open-access article distributed under the terms of the Creative Commons Attribution Non-Commercial License (http://creativecommons.org/ licenses/by-nc/4.0) which permits unrestricted noncommercial use, distribution, and reproduction in any medium, provided the original work is properly cited. Copyright ( 2019 by Korean Academy of Rehabilitation Medicine 


\section{INTRODUCTION}

To begin with, the numbers of patients with lower extremity osteoarthritis is increasing year by year. In this sense, it is known that lower limb osteoarthritis causes a decline in motor function and balance function in the patient affected with this condition, which can be responsible to leading to a variety of problems such as depression, metabolic syndrome, and flail due to a decrease in activities of daily living and quality of life $[1,2]$. These are major social problems and causes of increased medical and nursing care expenses in Japan, due to the requirement of long-term care for patients experiencing this condition [3]. Therefore, when the patient notes that the individual's osteoarthritis gets worse, a total joint replacement surgery is often recommended at that time $[4,5]$. However, even in the case of total joint replacement surgery, postoperative rehabilitation does not proceed smoothly in patients who have decreased mental and physical activity, which may result in a poor surgical outcome [6]. Therefore, for patients with lower extremity osteoarthritis, the necessity of maintaining and improving mental and physical functions before and after surgery has been advocated as a beneficial outcome for the patient $[7,8]$.

Anti-gravity treadmills can be used for training with a reduced load on the lower limbs and cardiopulmonary system by floating the body in a pneumatic chamber. Indeed, the study of Patil et al. [9] reported that the force applied to the knee on the anti-gravity treadmill decreased in patients after a total knee replacement surgery according to the amount of the noted lower extremity relief. Therefore, on an anti-gravity treadmill, walking and endurance training can be performed without exacerbation of pain by adjusting the load on the operated limb, as is the case even in patients early after joint replacement surgery $[10,11]$. The greatest feature of the anti-gravity treadmill is the breadth of adaptation even for those who have difficulty using an ordinary treadmill, and effects such as increased endurance, muscle strength, and ambulation ability are expected as a result using this method of rehabilitation $[12,13]$. The anti-gravity treadmill has fewer facility management problems than the use of pools, and it can be applied to various rehabilitation and training programs because nearly natural walking and running can be performed with a stable partial load. In- deed, Hambly et al. [14] conducted rehabilitation with an anti-gravity treadmill on postoperative patients with knee osteochondral defect, and reported that the knee function and self-efficacy improved with this therapy. In addition, Eastlack et al. [15] used an anti-gravity treadmill for patients early after anterior cruciate ligament (ACL) reconstruction and reported that pain was relieved and safety rehabilitation was achieved. On the anti-gravity treadmill, exercise intensity decreases due to a decrease in the load on the lower limbs, but it is possible to compensate for the decline in exercise intensity by increasing the walking speed $[16,17]$. Currently, Henkelmann et al. [18] is noted as having been performing an RCT using an anti-gravity treadmill in postoperative cases after fracture of the lower limbs, and it is expected that high-quality evidence will be obtained from the continued study of various diseases in the future.

In addition, in belt electrode-type skeletal muscle electrical stimulation (B-SES), especially AUTO TENS PRO (Homer Ion Corp., Tokyo, Japan), all of the belts are electrodes. As has been seen, with B-SES, muscle training can be done on a bed without leg joint motion. Therefore, it is determined that it is possible to safely carry out training on a bed even in cases where conventional rehabilitation is considered to be difficult, such as cases involving wound pain and restriction of range of motion in the early stages after ACL reconstruction, or in the associated case of a lower extremity total joint replacement surgery. Indeed, Hasegawa et al. [19] reported that 4 weeks of BSES training as early rehabilitation prevented muscle atrophy and muscle weakness after ACL reconstruction. In addition, Miyamoto et al. [20] reported that lower limb muscular strength increased with B-SES training in healthy subjects, and they also reported that endurance was improved by muscle stimulation in B-SES. Furthermore, Gremeaux et al. [21] reported that a lowfrequency electric stimulation method performed on the quadriceps and triceps triusis muscles of patients after total hip arthroplasty (THA) for 5 weeks, was known to have resulted in a greater improvement in knee extension muscle strength than with the use of conventional physical therapy. Thus, in recent years, the effects of B-SES on improving muscle strength and endurance have been clarified [22,23].

In this way, new physical therapy equipment has recently been developed and incorporated into postopera- 
tive rehabilitation for the benefit of patient outcomes. Therefore, we have devised and conducted a painless hybrid physiotherapy combining an anti-gravity treadmill and B-SES as an early rehabilitation program for patients after lower extremity total joint replacement surgery; however, the effects of this hybrid physiotherapy have not yet been clarified to date. In the present study, we aimed to verify the effects of hybrid physiotherapy combining an anti-gravity treadmill and B-SES on patients early after THA, as a lower limb total joint replacement for lower limb osteoarthritis retrospectively.

\section{MATERIALS AND METHODS}

\section{Patients}

This study was designed as a retrospective study. We retrospectively examined the methods and the outcomes of the postoperative rehabilitation, in the cases in which THA was performed for hip osteoarthritis in Hiroshima University Hospital from January 2017 to May 2018. In this case, the patients were assigned either to the conventional physical therapy group ('conventional group') or the hybrid physiotherapy group ('hybrid group'). The exclusion criteria included (1) cases who walking was difficult due to comorbidities before and after THA surgery, (2) cases where the patient could not speak Japanese, (3) cases where the patient consent form was not obtained due to psychiatric symptoms such as dementia, and (4) cases where the preoperative or postoperative evaluation could not be performed.

During the study period, there were 59 patients who underwent THA due to a diagnosis of hip osteoarthritis in these patients. Of these, 15 cases were excluded for the exclusion criteria ( 5 cases had difficulty walking before and after surgery due to comorbidities, 1 case could not speak Japanese, 2 cases did not provide consent to participate in the study, and 7 cases did not have preoperative or postoperative evaluation). In the leaving of 44 patients for analysis, there were 22 patients who underwent the conventional physical therapy (conventional group), and the other 22 patients underwent the hybrid physiotherapy (hybrid group). All subjects have undergone rehabilitation treatment for 2 weeks, and have received a postoperative evaluation. The demographic characteristics of the patients in both groups are shown in Table 1. It is noted that there were no differences were found between
Table 1. Demographic characteristics of patients in the conventional and hybrid group

\begin{tabular}{|c|c|c|}
\hline & $\begin{array}{l}\text { Conventional } \\
\text { group }(n=22)\end{array}$ & $\begin{array}{c}\text { Hybrid group } \\
(\mathbf{n}=22)\end{array}$ \\
\hline Age (yr) & $67.4 \pm 10.5$ & $64.4 \pm 11.2$ \\
\hline \multicolumn{3}{|l|}{ Gender } \\
\hline Male & 6 & 7 \\
\hline Female & 16 & 15 \\
\hline Body mass index $\left(\mathrm{kg} / \mathrm{m}^{2}\right)$ & $24.1 \pm 3.5$ & $25.4 \pm 4.3$ \\
\hline $\begin{array}{l}\text { Days from first visit to } \\
\text { operation (day) }\end{array}$ & $259.9 \pm 249.6$ & $249.7 \pm 241.1$ \\
\hline \multicolumn{3}{|l|}{ Side of operation } \\
\hline Right & 9 & 10 \\
\hline Left & 13 & 12 \\
\hline $\begin{array}{l}\text { Length of hospital stay } \\
\text { (day) }\end{array}$ & $19.4 \pm 4.5$ & $17.8 \pm 5.1$ \\
\hline Home return rate $(\%)$ & 68.1 & 86.4 \\
\hline
\end{tabular}

Values are presented as mean \pm standard deviation.

the groups in age, sex, body mass index, number of days from first visit to operation, and side of operation.

\section{Rehabilitation}

Fig. 1 shows the conventional THA postoperative rehabilitation protocol that was adopted in our hospital. From the first day after surgery, rehabilitation was started by a physiotherapist specializing in hip joint diseases, and rehabilitation was given every day during hospitalization. It is noted that from 1 day after surgery, hip range of motion exercises, muscle strength training on the operated side and walking exercises with the use of a walker were started with the patient. The T-cane walking exercise was permitted from the 7th postoperative day; however, only the hip joint rotation and bridging were prohibited during hospitalization. To begin with, during hospitalization, rehabilitation for about 30-40 minutes was conducted every day in the conventional group, consisting mainly of range of motion exercises in the lower limb joints, muscle strength training using weights and walking exercises according to the THA postoperative rehabilitation protocol. In the hybrid group, the same training as the conventional group was carried out for about 20 minutes, and training on an anti-gravity treadmill and B-SES training were added every day during hospitalization. It is noted that on anti-gravity treadmills, especially the AlterG (Alter-G Corp., Fremont, CA, USA), the load on the 
lower extremities can be reduced from $100 \%$ to as low as $20 \%$ in precise $1 \%$ increments. Also, since the patient is fixed to the treadmill with dedicated shorts, there is little danger of falling down during the process to increase safety during the testing (Fig. 2A). The load of the antigravity treadmill was set to such a degree that pain did

\begin{tabular}{|l|l|l|l|} 
& Day 1-day 6 & Day 7-day 13 & Day 14-day 21 \\
\hline $\begin{array}{l}\text { Non-operated leg } \\
\text { training }\end{array}$ & & & \\
\cline { 2 - 3 } $\begin{array}{l}\text { Hip range of motion } \\
\text { exercises, except } \\
\text { internal rotation }\end{array}$ & & & \\
\cline { 2 - 3 } $\begin{array}{l}\text { Muscle strength } \\
\text { training around hip } \\
\text { joint }\end{array}$ & & & \\
\hline $\begin{array}{l}\text { Straight leg raise } \\
\text { exercise }\end{array}$ & & \\
\hline $\begin{array}{l}\text { Walking exercise } \\
\text { with a walker }\end{array}$ & & \\
\hline $\begin{array}{l}\text { Walking exercise } \\
\text { with T-cane }\end{array}$ & & \\
\hline Discharge & & \\
\hline
\end{tabular}

Fig. 1. Conventional postoperative rehabilitation protocol for total hip arthroplasty operation in Hiroshima University Hospital. From one day after surgery, it is permitted for the patient to begin a range-of-motion exercise, muscle strength training on the operated side, and walking exercise with a walker. During this timeframe, a T-cane walking exercise is permitted from the 7th postoperative day. It is noted that only hip joint rotation and bridging are prohibited postoperatively during the hospitalization. not occur at the time of loading. In addition, the walking speed started from a comfortable pace and gradually increased in effort to a faster pace. The maximum walking speed was adjusted to reach a target heart rate calculated by Karvonen method ( $k=0.5)$ [24]. The walking time was set to 10 minutes a day. To begin with, the B-SES is a new, low frequency, electrical stimulation device that allows electricity to flow through the entire lower limb as a tube state, and all the muscles of the lower limbs can be moved by wrapping the belt around the legs (Fig. 2B). The stimulation condition by B-SES was performed for 10 minutes a day at a frequency of $20 \mathrm{~Hz}$, a pulse width of $250 \mu \mathrm{s}$, a duty cycle of 5 seconds stimulation plus 2 seconds pause, in accordance with Hasegawa et al. [19].

\section{Outcome measures}

In this study, the Numerical Rating Scale (NRS) for pain which rates pain on an 11-point scale from 0 to 10 , surgical side knee joint extension force, 10 -m walking test, Timed Up and Go test (TUG), and 6-minute walking distance (6MD) were measured. For measurements, the use of a cane was permitted, and $\mu$ Tas F1 (ANIMA Corp., Tokyo, Japan) was used for muscle strength measurement on the operated side of the knee joint.

\section{Length of hospital stay and home return rate}

The length of hospital stay and the home return rate were $19.4 \pm 4.5$ days and $68.1 \%$ in the conventional group and $17.8 \pm 5.1$ days and $86.4 \%$ in the hybrid group, respec-
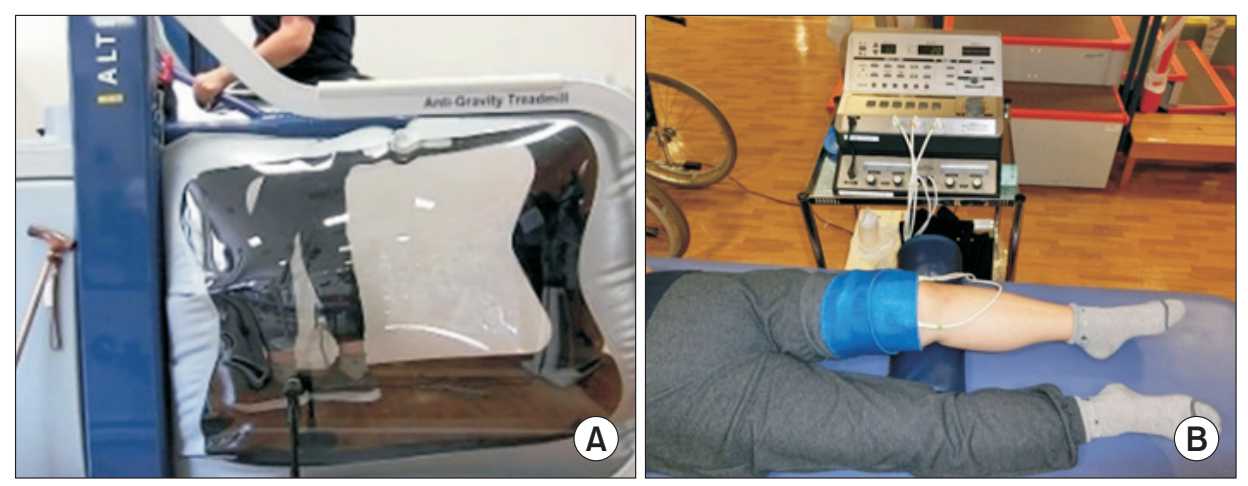

Fig. 2. New physical therapy equipment. (A) Anti-gravity treadmill. On the anti-gravity treadmill, especially AlterG (Alter-G Corp., Fremont, CA, USA), the load on the lower extremities can be adjusted every $1 \%$ within the range of $20 \%-100 \%$. (B) Belt electrode type skeletal muscle electrical stimulation (B-SES). In AUTO TENS PRO (Homer Ion Corp., Tokyo, Japan), all the belts are electrodes. It is known as a new electrical stimulation device that allows electricity to flow through the entire lower limb in a tube state, and moves all of the muscles of the lower limbs by wrapping the belt around the legs. 
tively (Table 1). In fact, there was no significant difference between the two groups in the length of hospital stay or the home return rate. However, the home return rate was slightly higher in the hybrid group than in the conventional group, although the difference was not considered to be significant.

\section{Statistical analysis}

In this study, the continuous variables were expressed as mean \pm standard deviation. In the comparison of preoperative and postoperative variables within the same group, a paired t-test was performed. In the comparison between the two groups, an unpaired t-test was used for continuous variables and a chi-square test was used for nominal variables (gender and side of operation). Generally speaking, for statistical processing, statistical software JMP pro version 13 (SAS Institute Inc., Cary, NC, USA) was used and $\mathrm{p}<0.05$ was regarded as significant in all analyses.

This study was approved by the Hiroshima University Epidemiological Research Ethics Review Committee (No. 1187).

\section{RESULTS}

In this study, early postoperative rehabilitation was performed safely in both the conventional and the hybrid group. To this end, there were no adverse events associated with rehabilitation, such as falls or respiratory and cardiovascular disorders were observed. The results of the preoperative and postoperative physical functions are shown in Table 2.

\section{Numerical Rating Scale}

IN this case, the preoperative NRS was $6.6 \pm 2.2$ in the conventional group and $6.3 \pm 2.2$ in the hybrid group, and there was no significant difference between the two groups. Additionally, the postoperative NRS was $2.0 \pm 1.6$ in the conventional group and $2.5 \pm 1.9$ in the hybrid group, both of which were significantly lower than before the operation; however, and no significant difference was observed between the two groups.

\section{0-m walking test}

The preoperative $10-\mathrm{m}$ walking test time was $11.5 \pm 5.3$ seconds in the conventional group and $10.7 \pm 4.4$ seconds in the hybrid group, and no significant difference was observed between the two groups. As has been seen, after surgery, the time in the conventional group was $13.9 \pm 6.4$ seconds and $12.3 \pm 3.9$ seconds in the hybrid group. In other words, there was no significant difference was observed between the two groups, and no significant difference was observed between preoperative and postoperative times in either group.

\section{Timed Up and Go test}

In this respect, the preoperative TUG was $12.9 \pm 5.6 \mathrm{sec}-$ onds in the conventional group and $12.1 \pm 5.5$ seconds in the hybrid group, and no significant difference was observed between the two groups. By all accounts, after surgery, the time in the conventional group was $15.5 \pm 6.1$ seconds and $14.4 \pm 6.3$ seconds in the hybrid group. Therefore, no significant difference was observed between the two groups, and no significant difference was observed between preoperative and postoperative TUG in either group.

Table 2. Preoperative and postoperative physical functions

\begin{tabular}{lccccc}
\hline & \multicolumn{2}{c}{ Conventional group } & & \multicolumn{2}{c}{ Hybrid group } \\
\cline { 2 - 3 } \cline { 5 - 6 } & Pre-operation & 2 weeks post-operation & & Pre-operation & 2 weeks post-operation \\
\hline NRS & $6.6 \pm 2.2$ & $2.0 \pm 1.6^{\text {a) }}$ & & $6.3 \pm 2.2$ & $2.5 \pm 1.9^{\text {a) }}$ \\
10-m walking test (s) & $11.5 \pm 5.3$ & $13.9 \pm 6.4$ & & $10.7 \pm 4.4$ & $12.3 \pm 3.9$ \\
TUG (s) & $12.9 \pm 5.6$ & $15.5 \pm 6.1$ & & $12.1 \pm 5.5$ & $14.4 \pm 6.3$ \\
Knee extension strength (kgf) & $17.8 \pm 3.7$ & $12.8 \pm 3.2^{\text {a) }}$ & & $18.1 \pm 7.3$ & $16.2 \pm 6.8^{\text {b) }}$ \\
6MD (m) & $294.1 \pm 114.4$ & $225.8 \pm 65.7^{\text {a) }}$ & & $307.0 \pm 134.1$ & $316.4 \pm 115.1^{\text {b) }}$
\end{tabular}

Values are presented as mean \pm standard deviation.

NRS, Numerical Rating Scale for pain; TUG, Timed Up and Go test; 6MD, 6-minute walking distance.

${ }^{a)} \mathrm{p}<0.05$ for pre-operation vs. 2 weeks post-operation.

${ }^{b)} \mathrm{p}<0.05$ for the hybrid group vs. conventional group. 


\section{Knee extension strength}

In this case, prior to surgery, the knee extension strength on the operated side was $17.8 \pm 3.7 \mathrm{kgf}$ in the conventional group and $18.1 \pm 7.3 \mathrm{kgf}$ in the hybrid group, and no significant difference was observed between the two groups. By the same token, it is noted that after surgery, knee extension strength in the conventional group $(12.8 \pm 3.2 \mathrm{kgf})$ was significantly lower than in the hybrid group (16.2 $\pm 6.8 \mathrm{kgf})$. In the preoperative and postoperative comparisons, no significant difference was observed in the hybrid group, whereas the conventional group showed a significant postoperative decrease in knee extension strength.

\section{6-minute walking distance}

The preoperative $6 \mathrm{MD}$ was $294.1 \pm 114.4 \mathrm{~m}$ in the conventional group and $307 \pm 134.1 \mathrm{~m}$ in the hybrid group, and no significant difference was recognized between these groups. After surgery, 6MD in the conventional group $(225.8 \pm 65.7 \mathrm{~m})$ was significantly shorter than in the hybrid group (316.4 $\pm 115.1 \mathrm{~m})$. Broadly speaking, in the preoperative and postoperative comparisons, no significant difference was observed in the hybrid group, whereas the conventional group showed a significant postoperative decrease in 6MD.

\section{DISCUSSION}

Recently, new physical therapy equipment has been developed and is beginning to be applied in postoperative rehabilitation for the benefit of patients post operatively. In fact, muscle strength training, walking and endurance exercises are important for early rehabilitation in patients after THA surgery, in addition to range of motion training. Therefore, considering the potential effects of antigravity treadmills and B-SES, we devised and conducted hybrid physiotherapy which worked as combining both an anti-gravity treadmill and B-SES training. However, to the best of our knowledge, our report on hybrid physiotherapy is the first study to use a combination of an antigravity treadmill and B-SES for postoperative rehabilitation to date. In our postoperative THA protocol, on the first day after surgery, the beginning of the lower limb muscle strength training on the operated side and walking training were permitted, but in reality, because of the injury pain experienced by the patient and the decline in physical and mental functions before the surgery, aggressive lower limb muscle strength training, walking and endurance training were noted as being difficult to achieve in that case. Therefore, in the conventional group, we started with range of motion exercises on a platform and muscle strength training using weights, and gradually advanced the course of rehabilitation to include walking training. The greatest characteristic of the hybrid physiotherapy we devised is that we can reduce the burden on the lower limbs and the cardiopulmonary system, and that the training can begin soon after the operation. In fact, in the hybrid group in the present study, it was possible to introduce muscle strength training, walking and endurance training just 1 day after THA.

Regarding walking ability, the times for the 10 -m walking test and TUG were slightly prolonged in both groups at 2 weeks after the operation compared as with before the operation, but the difference was not considered to be significant. In addition, there was no significant difference between the two groups at 2 weeks after the operation, and thus, it was noted that the short distance walking ability was equally improved to the preoperative level in both groups.

In the conventional group, knee extension muscle strength and 6MD at 2 weeks after the operation were significantly lower than those before surgery. However, in the hybrid group, those values noted at 2 weeks after the operation were not significantly lower than those that were measured before the surgery. Also, at 2 weeks after the operation, there was a significant difference between the values of knee extension muscle strength and 6MD in the hybrid group, and those who were in the conventional group. Therefore, in the conventional group, it is noted that the lower limb muscular strength and endurance declined early after surgery, but in the hybrid group, the measured lower limb muscular strength and endurance were preserved and improved up to the preoperative level. The question then follows from the above data that the effects of maintaining and increasing lower limb muscular strength and endurance through hybrid physiotherapy as an early rehabilitation program for patients after THA was in this case clarified.

Like many Japanese acute phase hospitals, our hospital adopts the Diagnosis Procedure Combination system [25], whereby the maximal length of hospital stay is generally 2-3 weeks. Therefore, if it is difficult to return home even 
3 weeks after surgery, the patient will be transferred to a rehabilitation hospital and continue rehabilitation for a maximal time frame of 3 months to return home [26]. In the present study, there was no significant difference in the length of hospital stay or in the home return rate between the two groups; however, more patients in the conventional group were transferred to a rehabilitation hospital, as compared with the hybrid group. One reason for this result is that the muscular strength and endurance were not sufficiently recovered in the participants of the conventional group.

This study had some limitations, which are noted below. First, the average rehabilitation intervention time per day was not noted as being completely consistent between the conventional group and the hybrid group. The time required for rehabilitation in the hybrid group was inevitably long, but in the conventional method, aggressive early rehabilitation cannot be performed for a long time, due to the patient's wound pain and the noted mental and physical function deterioration. Secondly, the intervention was carried out by several therapists instead of the situation of having only the therapist in charge; however, the basic rehabilitation program was noted as being essentially the same. In addition, the intervention was done with a doctor's supervision, and it seemed that there were almost no differences to be recorded between the utilized therapists. Thirdly, in the present study, the load of the anti-gravity treadmill varied among the noted participant cases. This research did not strictly consider the effects of the anti-gravity treadmill alone. Also, since it is important that the patient continue the training, the amount of the load was not fixed. It is important that endurance exercises on the anti-gravity treadmill start on the earliest postoperative day possible, which is understood to be a difficult endeavor with the use of conventional methods. Fourthly, we initially considered preparing an early rehabilitation program for patients after lower extremity total joint replacement for osteoarthritis. Therefore, in that scenario we initially considered including patients after total knee arthroplasty as well. However, B-SES is already routinely used in patients after total knee arthroplasty, and establishing a control group for hybrid physiotherapy is an ethical problem. Fifth, due to restrictions that were imposed on the hospitalization period in Japan, the intervention period was only 2 weeks after surgery. Many of the present patients resided in distant areas, and follow-up observations after discharge from the hospital were conducted by doctors closer to the patient's homes. Therefore, it was impossible to conduct long-term evaluation of the participants after surgery. Finally, this study did not compare the effects of conventional methods and the anti-gravity treadmill and/or BSES hybrid physiotherapy. In the future, a large-scale multicenter study is necessary to compare the short-term and long-term effects among the patient's use of the antigravity treadmill alone, B-SES alone and with the use of hybrid physiotherapy.

In conclusion, with the use of hybrid physiotherapy, muscle strength, walking and endurance training were possible immediately after surgery. In the conventional group, lower limb muscular strength and endurance declined early after THA; however, these functions were maintained in the hybrid group. Therefore, it was suggested that hybrid physiotherapy could maintain the patient's physical function early after THA.

\section{CONFLICT OF INTEREST}

No potential conflict of interest relevant to this article was reported.

\section{ACKNOWLEDGMENTS}

This work was supported by JSPS KAKENHI Grant-inAid for Scientific Research C (No. JP 15K01369).

\section{AUTHOR CONTRIBUTION}

Conceptualization: Mikami Y, Orita N, Yamasaki T. Methodology: Mikami Y, Adachi N. Formal analysis: Kimura H. Funding acquisition: Orita N, Yamasaki T. Project administration: Mikami Y, Orita N, Yamasaki T. Visualization: Kamijo Y. Writing - original draft: Mikami Y, Kamijo Y. Writing - review and editing: Adachi N. Approval of final manuscript: all authors.

\section{REFERENCES}

1. Kondo K, Jingushi S, Ohfuji S, Sofue M, Itoman M, Matsumoto T, et al. Factors associated with functional limitations in the daily living activities of Japanese hip osteoarthritis patients. Int J Rheum Dis 2017;20:1372- 
82.

2. Bobic Lucic L, Grazio S. Impact of balance confidence on daily living activities of older people with knee osteoarthritis with regard to balance, physical function, pain, and quality of life: a preliminary report. Clin Gerontol 2018;41:357-65.

3. Akune T, Muraki S, Oka H, Tanaka S, Kawaguchi H, Tokimura $\mathrm{F}$, et al. Incidence of certified need of care in the long-term care insurance system and its risk factors in the elderly of Japanese population-based cohorts: the ROAD study. Geriatr Gerontol Int 2014;14:695-701.

4. Fujita K, Makimoto K, Tanaka R, Mawatari M, Hotokebuchi T. Prospective study of physical activity and quality of life in Japanese women undergoing total hip arthroplasty. J Orthop Sci 2013;18:45-53.

5. Maeda Y, Nakamura N, Sugano N. Improvement of activities of daily living after total hip arthroplasty using a computed tomography-based navigation system. J Artif Organs 2017;20:152-7.

6. Fortin PR, Clarke AE, Joseph L, Liang MH, Tanzer $\mathrm{M}$, Ferland D, et al. Outcomes of total hip and knee replacement: preoperative functional status predicts outcomes at six months after surgery. Arthritis Rheum 1999;42:1722-8.

7. Wallis JA, Taylor NF. Pre-operative interventions (nonsurgical and non-pharmacological) for patients with hip or knee osteoarthritis awaiting joint replacement surgery: a systematic review and meta-analysis. Osteoarthritis Cartilage 2011;19:1381-95.

8. Coudeyre E, Jardin C, Givron P, Ribinik P, Revel M, Rannou F. Could preoperative rehabilitation modify postoperative outcomes after total hip and knee arthroplasty? Elaboration of French clinical practice guidelines. Ann Readapt Med Phys 2007;50:189-97.

9. Patil S, Steklov N, Bugbee WD, Goldberg T, Colwell CW Jr, D'Lima DD. Anti-gravity treadmills are effective in reducing knee forces. J Orthop Res 2013;31:672-9.

10. Bugbee WD, Pulido PA, Goldberg T, D'Lima DD. Use of an anti-gravity treadmill for early postoperative rehabilitation after total knee replacement: a pilot study to determine safety and feasibility. Am J Orthop (Belle Mead NJ) 2016;45:E167-73.

11. Huang CH, Schroeder ET, Powers C. Antigravity treadmill training during the early rehabilitation phase following unicompartmental knee arthroplasty: a case series. Physiother Theory Pract 2019;35:489-94.
12. Mikami Y, Fukuhara K, Kawae T, Kimura H, Ochi M. The effect of anti-gravity treadmill training for prosthetic rehabilitation of a case with below-knee amputation. Prosthet Orthot Int 2015;39:502-6.

13. Cutuk A, Groppo ER, Quigley EJ, White KW, Pedowitz RA, Hargens AR. Ambulation in simulated fractional gravity using lower body positive pressure: cardiovascular safety and gait analyses. J Appl Physiol (1985) 2006;101:771-7.

14. Hambly K, Poomsalood S, Mundy E. Return to running following knee osteochondral repair using an antigravity treadmill: a case report. Phys Ther Sport 2017; 26:35-40.

15. Eastlack RK, Hargens AR, Groppo ER, Steinbach GC, White KK, Pedowitz RA. Lower body positive-pressure exercise after knee surgery. Clin Orthop Relat Res 2005; (431):213-9.

16. Kawae T, Mikami Y, Fukuhara K, Kimura H, Adachi N. Anti-gravity treadmill can promote aerobic exercise for lower limb osteoarthritis patients. J Phys Ther Sci 2017; 29:1444-8.

17. Grabowski AM, Kram R. Effects of velocity and weight support on ground reaction forces and metabolic power during running. J Appl Biomech 2008;24:28897.

18. Henkelmann R, Schneider S, Muller D, Gahr R, Josten $\mathrm{C}$, Bohme J. Outcome of patients after lower limb fracture with partial weight bearing postoperatively treated with or without anti-gravity treadmill (alter $\mathrm{G}^{\circledR}$ ) during six weeks of rehabilitation: a protocol of a prospective randomized trial. BMC Musculoskelet Disord 2017;18:104.

19. Hasegawa S, Kobayashi M, Arai R, Tamaki A, Nakamura T, Moritani T. Effect of early implementation of electrical muscle stimulation to prevent muscle atrophy and weakness in patients after anterior cruciate ligament reconstruction. J Electromyogr Kinesiol 2011;21:622-30.

20. Miyamoto T, Kamada H, Tamaki A, Moritani T. Lowintensity electrical muscle stimulation induces significant increases in muscle strength and cardiorespiratory fitness. Eur J Sport Sci 2016;16:1104-10.

21. Gremeaux V, Renault J, Pardon L, Deley G, Lepers R, Casillas JM. Low-frequency electric muscle stimulation combined with physical therapy after total hip arthroplasty for hip osteoarthritis in elderly patients: 
a randomized controlled trial. Arch Phys Med Rehabil 2008;89:2265-73.

22. Nishida MM, Tsuboyama T, Moritani T, Arai H. Review of the evidence on the use of electrical muscle stimulation to treat sarcopenia. Eur Geriatr Med 2016;7:26771.

23. Numata $H$, Nakase J, Inaki $A$, Mochizuki $T$, Oshima T, Takata Y, et al. Effects of the belt electrode skeletal muscle electrical stimulation system on lower extremity skeletal muscle activity: evaluation using positron emission tomography. J Orthop Sci 2016;21:53-6.

24. Karvonen MJ, Kentala E, Mustala O. The effects of training on heart rate; a longitudinal study. Ann Med Exp Biol Fenn 1957;35:307-15.

25. Nakagawa Y, Takemura T, Yoshihara H, Nakagawa Y. A new accounting system for financial balance based on personnel cost after the introduction of a DPC/DRG system. J Med Syst 2011;35:251-64.

26. Shiraishi N, Suzuki Y, Matsumoto D, Jeong S, Sugiyama M, Kondo K, et al. The effect of additional training on motor outcomes at discharge from recovery phase rehabilitation wards: a survey from multi-center stroke data bank in Japan. PLoS One 2014;9:e91738. 